\title{
Colloid Thin-Layer Chromatography: A Tool for Gold Sols Validation before Used in Application
}

\author{
Jingjie Luo1,2, Caroline Bertagnolli1,3*, Pierre Petit ${ }^{4}$, Corinne Petit ${ }^{1}$ \\ ${ }^{1}$ Université de Strasbourg, CNRS, Institut de Chimie et Procédés pour l'Energie, l'Environnement et la Santé, Strasbourg, France \\ ${ }^{2}$ Lab of Advanced Materials \& Catalytic Engineering (AM \& CE), School of Petroleum and Chemical Engineering, Dalian \\ University of Technology, Panjin, China \\ ${ }^{3}$ Université de Strasbourg, CNRS, Institut Pluridisciplinaire Hubert Curien, Strasbourg, France \\ ${ }^{4}$ Université de Strasbourg, CNRS, Institut Charles SADRON, Strasbourg, France \\ Email: *caroline.bertagnolli@unistra.fr
}

How to cite this paper: Luo, J.J., Bertagnolli, C., Petit, P. and Petit, C. (2021) Colloid Thin-Layer Chromatography: A Tool for Gold Sols Validation before Used in Application. Journal of Materials Science and Chemical Engineering, 9, 22-36. https://doi.org/10.4236/msce.2021.99003

Received: August 25, 2021

Accepted: September 23, 2021

Published: September 26, 2021

Copyright $\odot 2021$ by author(s) and Scientific Research Publishing Inc. This work is licensed under the Creative Commons Attribution International License (CC BY 4.0).

http://creativecommons.org/licenses/by/4.0/

\begin{abstract}
Gold nanoparticles have been increasingly used in catalysis, biomedical imaging, biological and chemical sensing, drug delivery, etc. In this study, a straightforward method that allows one to monitor the synthesis of gold sols and their aging, before their fine characterization by sophisticated techniques and before their use is described. Indeed, the "Colloid Thin-Layer Chromatography" method allows one to check the quality of gold colloidal sols during the synthesis. It is also well adapted for monitoring the aging of the sol before the visual observation of its degradation.
\end{abstract}

\section{Keywords}

Gold, Colloid, Nanoparticle, Aggregation, Turkevich

\section{Introduction}

Gold nanoparticles (GNPs) as silver and copper ones exhibit Surface Plasmon Resonance (SPR) given vibrant colors produced by their interaction with visible light [1]. The unique optical-electronics properties of GNPs have been used for smart technological applications such as organic photovoltaic cells, sensors, drug delivery [2], electronics and catalysis.

Recently, Zhao et al. [3] and De Souza et al. [4] listed the methods used to obtain GNPs. These two reviews focus on synthesis pathways by chemical reduction. The most frequently cited is Turkevich's method, simple and fast, using the citrate function as the reducer and stabilizer. Many methods are derived from it, and, depending on the synthetic route, monodispersed GNPs of various sizes 
ranging between 4 and $186 \mathrm{~nm}$ are obtained.

The techniques commonly used for the characterization of shape and size distributions of colloid sols are Dynamic Light Scattering (DLS), UV-vis spectroscopy and microscopies. DLS and UV-Vis are practical techniques for the rapid characterization of colloid sols. Microscopy techniques such as Transmission Electron Microscopy (TEM) and Scanning Electron Microscopy (SEM) are used for the fine characterization of the size distribution of GNPs. However, these sols evolve with time and aggregation of GNPs occurs before the visual observation of the sols degradation in the form of a black suspension, identified as filaments of intertwined nanoparticles [5], which eventually precipitate. Thus, to be rigorous, before each use of such GNPs sols, they need to be characterized again to control their quality, although it costs time and human resources [6] [7] [8].

Thin-Layer Chromatography (TLC) is a universal chromatography technique used to separate non-volatile mixtures. It consists of a plate made of a thin layer of silica gel deposited on an aluminum or glass sheet. When dipping the bottom of a plate into a mixture, the solvent is drawn up into the plate via capillary action. It allows one to carry out almost every type of separation by suitably choosing the mobile phase. In this article, we present the applicability of TLC (hereafter named "Colloid Thin-Layer Chromatography"-CTLC) to follow the states of GNPs sols in water. The GNP's sol imprint on the TLC is time stable. Then, once a GNPs sol is freshly prepared, its imprint is usable as a reference. Thus, before using a sol after having to store it, the comparison between its CTLC and the reference sample ensures that it is suitable or not for further investigations. This work reports a detailed study about the pertinence of CTLC on GNPs sols prepared by Turkevitch's method.

\section{Experimental}

Trisodium citrate dihydrate $\left(\mathrm{HOC}(\mathrm{COONa})\left(\mathrm{CH}_{2} \mathrm{COONa}\right)_{2} \cdot 2 \mathrm{H}_{2} \mathrm{O}\right.$, Sigma Aldrich, $\geq 99 \%$ ), tetrachloroauric acid ( $\mathrm{HAuCl}_{4}$, Aldrich, $99.99 \%$ purity), hydrochloric acid ( $\mathrm{HCl}, \mathrm{ACS}$ reagent, 37\%), sodium hydroxide $(\mathrm{NaOH}, \mathrm{ACS}$ reagent, $97 \%$ purity, pellets), citric acid $\left(\mathrm{C}_{6} \mathrm{H}_{8} \mathrm{O}_{7} \cdot \mathrm{H}_{2} \mathrm{O}\right.$, Acros, 99.5\%) and sodium chloride $(\mathrm{NaCl}$, Aldrich, 99\% purity) were used in this work.

\subsection{Synthesis of Colloidal GNPs}

GNPs sol syntheses were based on the classical Turkevich's method. All glass vessels used in the following procedures were cleaned with aqua regia and rinsed thoroughly in Milli-Q grade water before use. $100 \mathrm{~mL}$ of Milli-Q water was stirred $(500 \mathrm{rpm})$ and was heated to its boiling temperature $\left(100^{\circ} \mathrm{C}\right)$ under reflux. $0.2 \mathrm{~mL}$ of trisodium citrate solution $0.5 \mathrm{~mol} \cdot \mathrm{L}^{-1}$ (as reducing and stabilizing agent) and $4 \mathrm{~mL}$ of tetrachloroauric acid $\left(\mathrm{HAuCl}_{4}\right)$ solution $0.1 \mathrm{mmol}$ $\mathrm{Au} \cdot \mathrm{L}^{-1}$ were added and the solution was agitated for 60 minutes. The intrinsic size of gold nanoparticles is measured at $30 \mathrm{~nm}$ by Transmission Electron Microscopy. 


\subsection{Aggregation/Agglomeration of the Sols}

Aggregation/agglomeration of colloidal nanoparticles may be induced by a change in the $\mathrm{pH}$, temperature or ionic state of their surrounding medium. Time aging was studied on sols after a long storage ( 6 and 24 months in the dark at $\left.4^{\circ} \mathrm{C}\right)$. The $\mathrm{pH}$ effects were investigated by acidic and basic treatments $(0.1 \mathrm{~mL}$ of $\mathrm{HCl}\left(0.12\right.$ or $\left.1.2 \mathrm{~mol} \cdot \mathrm{L}^{-1}\right)$; citric acid $\left(0.1\right.$ or $\left.1 \mathrm{~mol} \cdot \mathrm{L}^{-1}\right)$; or $\mathrm{NaOH}(0.25$ or 2.5 $\left.\mathrm{mol} \cdot \mathrm{L}^{-1}\right)$ solution in $1 \mathrm{~mL}$ of gold sols). The ionic strength effect was studied by addition $\mathrm{NaCl}\left(0.1 \mathrm{~mL}\right.$ of $\mathrm{NaCl}\left(1 \mathrm{~mol} \cdot \mathrm{L}^{-1}\right)$ in $1 \mathrm{~mL}$ of gold sols $)$ and finally the temperature effect by heating the GNPs sols during $5 \mathrm{~h}$ under reflux at $100^{\circ} \mathrm{C}$.

\subsection{Characterization of Colloidal GNPs}

Photographs of the deposition of gold sols were obtained by Drop Shape Krüss (Drop shape analyzer DSA25). UV-vis absorption spectra were performed in the range of $300-800 \mathrm{~nm}$ (Perkin Elmer-Lambda 25 spectrometer) by using a quartz cell. Dynamic Light Scattering characterizations were performed using a Malvern Zetasizer (Nano ZS) in dual mode $\left(173^{\circ}\right.$ and $13^{\circ}$ ) [9]. The parameters used for DLS characterization are: $25^{\circ} \mathrm{C}$, viscosity $0.8872 \mathrm{cP}$, material absorption 3.32 , refractive index 0.2 , measurement duration extend duration for large particles $\times 1000$. The reported relative intensities $\left(I / I_{0}\right)$ have been determined by fitting the experimental curves with Gaussian fits. Transmission Electron Microscopy (TEM) imaging was carried out using a Technai G2 microscope (FEI) at $200 \mathrm{kV}$. Images were acquired with an Eagle2K ssCCD camera (FEI), five microliters of the gold colloidal sol were deposited onto a freshly glow discharged carbon-covered copper grid (400 mesh). The suspension was left for $2 \mathrm{~min}$ and finally dried at room temperature using filter paper. Scanning Electron Microscopy (SEM) analyses were carried out on a JEOL 6700F microscope working at $10 \mathrm{kV}$ accelerated voltage.

\subsection{Colloid Thin-Layer Chromatography (CTLC)}

The CTLC test was made on a thin layer of silica gel TLC Silica Gel 60 F254 from Merck, often used for following organic reactions. Specifications of the TLC silica gel are given in Table 1. In our case, the use of TLC is different from the one used in organic chemistry. One droplet $(20 \mu \mathrm{L})$ of the colloid sol was deposited onto a dry TLC plate lying flat on the bench.

Table 1. TLC specifications (Merck KGaA)

\begin{tabular}{cc}
\hline Material & $\begin{array}{c}\text { aluminum support; silica gel } 60 \text { matrix; } \\
\text { fluorescent indicator }\end{array}$ \\
\hline Layer thickness & $210-270 \mu \mathrm{m}$ \\
d 50 (laser diffraction, size distribution) & $9.5-11.5 \mu \mathrm{m}$ \\
Pore volume $\left(\mathrm{N}_{2}\right.$-isotherm) & $0.74-0.84 \mathrm{~mL} \cdot \mathrm{g}^{-1}$ \\
Specific surface area & $480-540 \mathrm{~m}^{2} \cdot \mathrm{g}^{-1}$ \\
(according to BET; 5-Pt. measurement) & \\
\hline
\end{tabular}




\section{Results and Discussion}

Typical CTLC test and terminology are reported in Figure 1. The first step is the contact between the droplet and silica gel on an area defined by the volume of the droplet. The deposition of the droplet causes the formation of an initial wet area named "initial contact zone". The initial contact zone of the deposited droplet has a diameter of about $0.5 \mathrm{~cm}$ for a droplet of $20 \mu \mathrm{L}$. With time, the diffusion of the solution is observed and a "moist-dry boundary" is formed at the interface between the wet area and the rest of the dry plate. The final observation of the plate is possible after complete drying, about 30 minutes.

The CTLC test of GNPs sol at different times of the synthesis was realized and presented on the top of Figure 2. One droplet is deposited on CTLC and once the plate is dry, a uniformly weakly colored disk limited by a single circular dark red-violet colored boundary (diameter of about $1 \mathrm{~cm}$ for a droplet of $20 \mu \mathrm{L}$ ) is observed whatever the reaction time. This can be explained by the fact that, after the droplet deposition, the free particles present in the solution are moving by diffusion of the solvent. During this process, they distribute uniformly within the volume of the layer of the silica gel, most of them being in the solvent to finally accumulate at the moist-dry boundary. This analysis confirms that the solution is indeed a colloidal suspension of gold nanoparticles.

The UV-vis spectra of the kinetics of GNPs formation are shown at the bottom of Figure 2. After one minute of reaction, the solution displays a grey color, with a corresponding UV-vis spectrum centered at $532 \mathrm{~nm}$. For larger times of

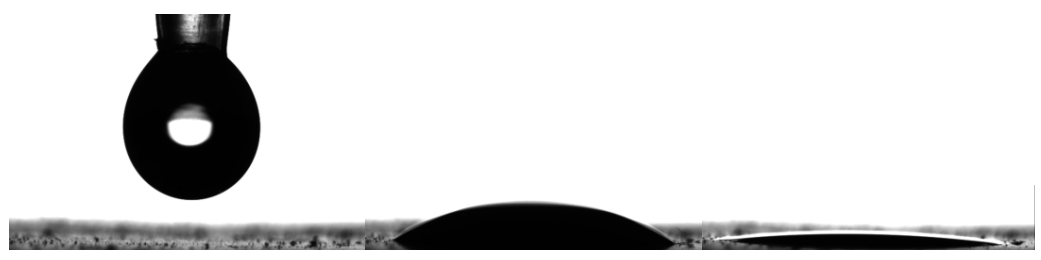

(a)

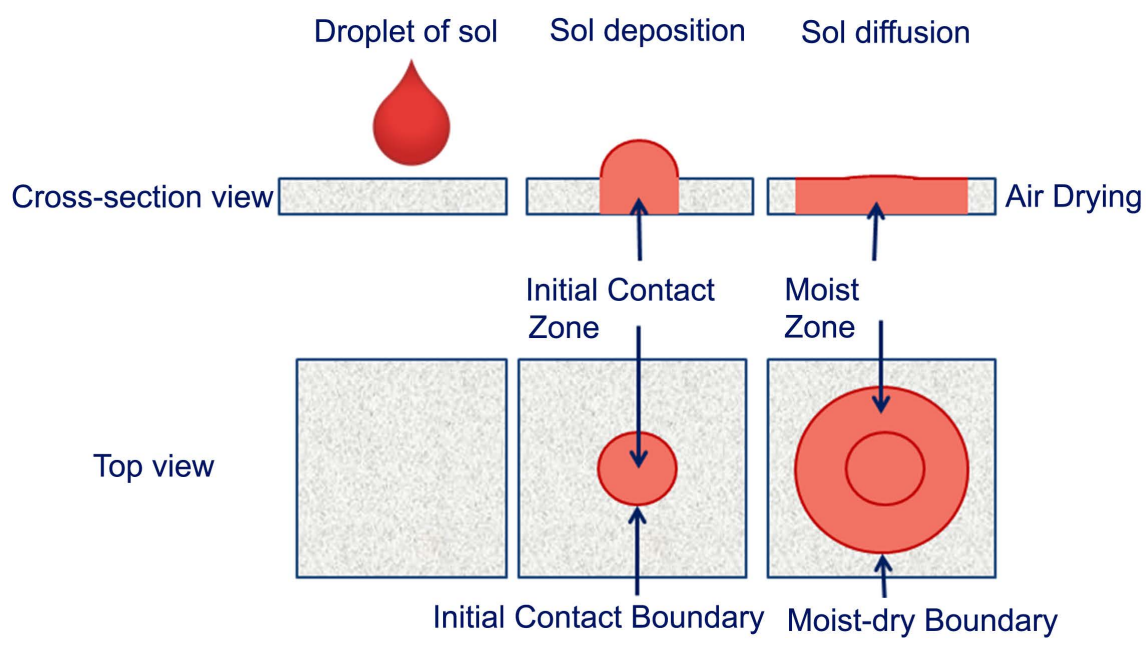

(b)

Figure 1. (a) Photography and (b) schematic illustration of typical CTLC test. 


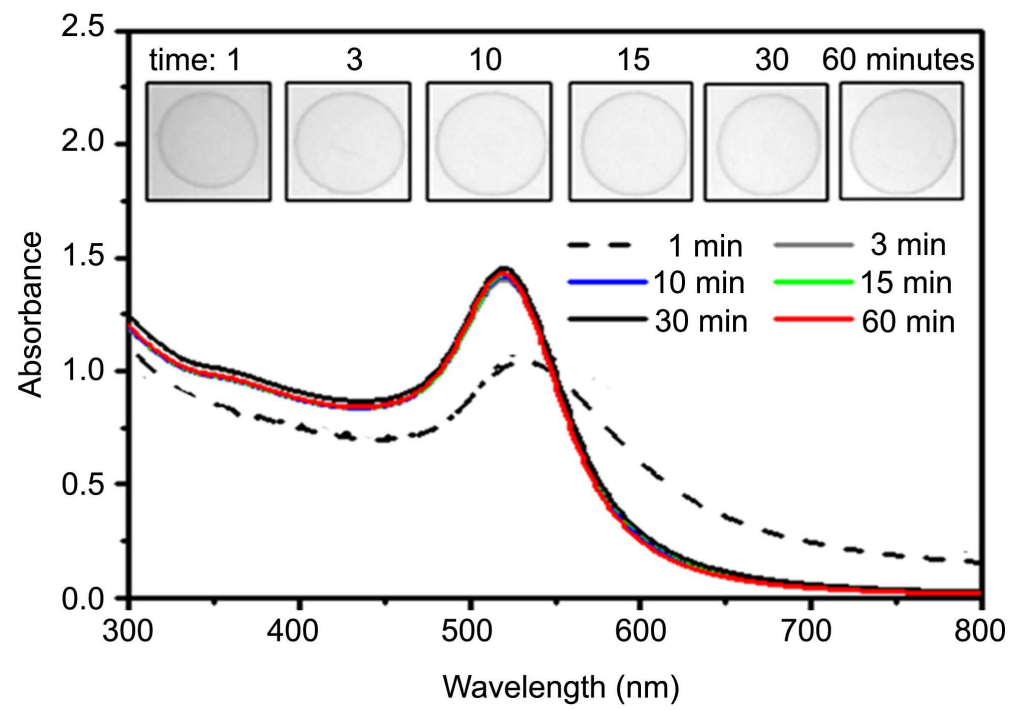

Figure 2. Images of TLC plates and UV-vis spectra in function of the reaction time of gold nanoparticles.

reaction, all GNPs sols display a wine-red color and a single absorption peak at $519 \mathrm{~nm}$ characteristic of spherical and small nanoparticles [10] [11]. The high citrate amount allows a quick reduction $(<3 \mathrm{~min})$ and then the stabilization of the gold nanoparticles by the citrate salt. These observations are in full agreement with those already reported [12]. A representation of protected gold nanoparticles in the sol during the GNPs formation, is presented in Scheme 1.

Citrate protection may be effected either by a change in the $\mathrm{pH}$, temperature or ionic state of their surrounding medium.

Figure 3(a) displays the results of CTLC tests of GNPs sol freshly prepared and after modification of its $\mathrm{pH}$ by addition of acid or base. For a $\mathrm{pH}$ modification at 3 or 10, GNPs sol does not change in color or aspect and shows the same CTLC profile as the freshly prepared one: one single circle at the moist-dry boundary. This indicates that the layer of citrate continues to protect the gold nanoparticles and the sol remains stable under these conditions, the corresponding state of gold nanoparticles and citrate species is presented in Scheme 2.

At pH 1 and pH 12 the link between citrate and gold was completely broken thus aggregation takes place and the color of the GNPs sol changes (from red to grey). The corresponding representation is given in Scheme 3. Due to the aggregation, GNPs are not available to migrate to the moist boundary of the TLC plate and a single circle (diameter $0.5 \mathrm{~cm}$ for a droplet of $20 \mu \mathrm{L}$ ) at the initial contact boundary is presented on the CTLC (Figure $3(\mathrm{a}), \mathrm{pH}=1$ by $\mathrm{HCl}$ addition and $\mathrm{pH}=12$ by $\mathrm{NaOH}$ addition). Grys et al. [13] showed that the citrate coordination is mostly bidentate and controlled by its protonation state. At $\mathrm{pH}$ 10 or 12 , which is considerably higher than the three $\mathrm{pKa}$ values of sodium citrate $\left(\mathrm{pKa}_{1}=3.14, \mathrm{pKa}_{2}=4.75\right.$ and $\left.\mathrm{pKa}_{3}=6.39\right)$, all carboxylate groups are deprotonated [14]. $\mathrm{NaOH}$ addition increases the interaction between sodium cations and carboxylate sites from the citrate protection layer (Scheme 2(b) and 


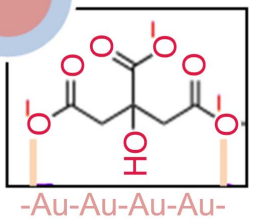

Scheme 1. Representation of gold nanoparticles freshly prepared (pink color) surrounded by citrates (blue color) and the carboxylate functions involved in the protection of the nanoparticles.

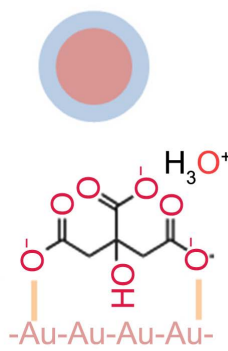

(a)

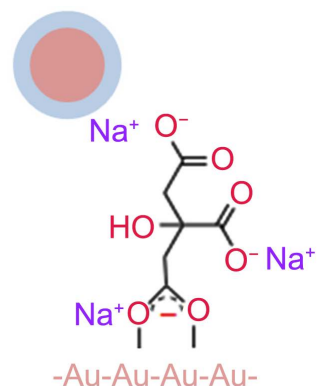

(b)

Scheme 2. Representation of a gold nanoparticle (pink color) surrounded by citrates (blue color) and the carboxylate functions involved after modification of $\mathrm{pH}$ by addition of (a) $\mathrm{HCl}, \mathrm{pH}=3$ or (b) $\mathrm{NaOH}, \mathrm{pH}=10$.

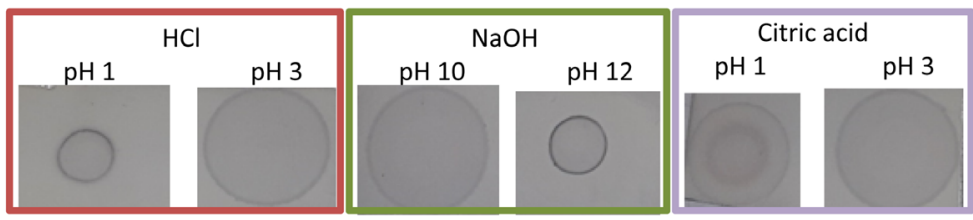

(a)

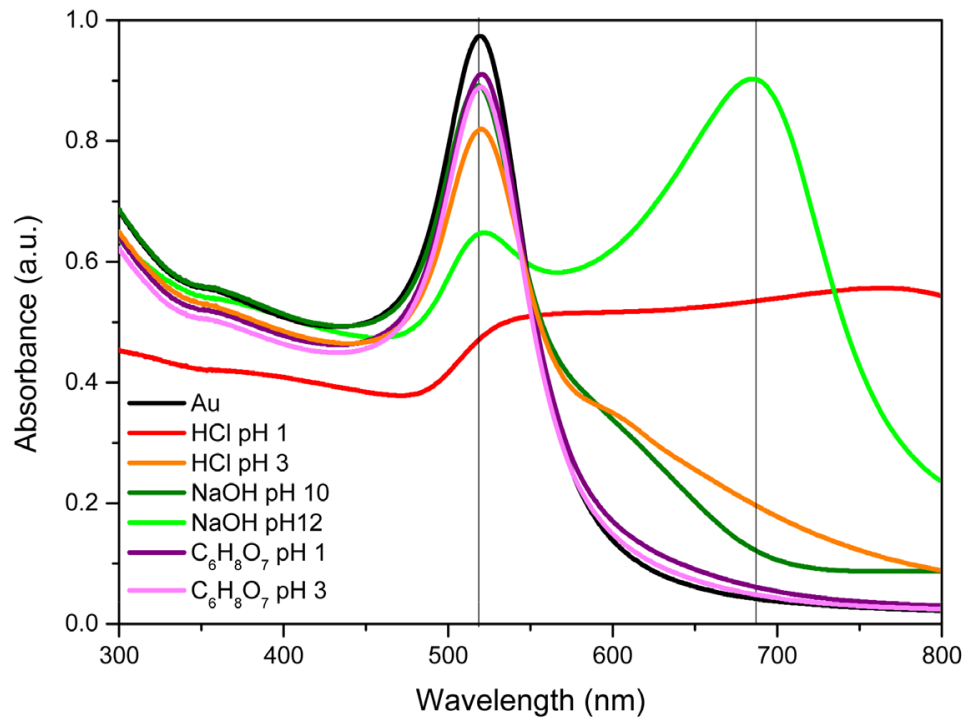

(b)

Figure 3. (a) CTLC tests of GNPs sol freshly prepared and after modification of its $\mathrm{pH}$ by addition of acid or base. (b) UV-vis spectra of gold sols after acid or base additions. 


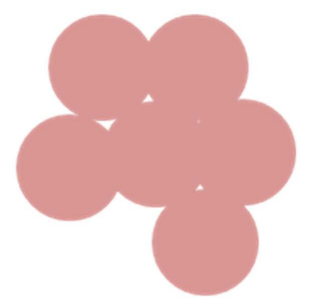<smiles>O=C(O)CC(O)(CC(=O)O)C(=O)O</smiles>

(a)

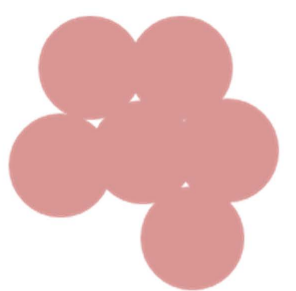<smiles>[NH3+][OH+]C(=O)CC(O)(CC([NH3+])=O)C(=O)[O-]</smiles>

(b)

Scheme 3. Representation of a gold nanoparticles (pink color) aggregation and the citric acid or the trisodium citrate form involved after modification of $\mathrm{pH}$ by addition of (a) $\mathrm{HCl}, \mathrm{pH}=1$ or (b) or (b) $\mathrm{NaOH}, \mathrm{pH}=12$.

Scheme 3(b)). Similar behavior may explain the CTLC profile for acid conditions, the total protonation of carboxylate sites promotes the aggregation at $\mathrm{pH}$ $=1$.

The addition of citric acid favors a special case with the creation of a double layer of citrate which forms on the free functions between the citrates fixed to the gold and the citric acid contained in the aqueous phase of the sol citrate as presented in Scheme 4. With a moderate addition of citric acid, the sol remains stable due to the repulsion of nanoparticles loaded with double layers of citrate. As the acid concentration increases, the $\mathrm{pH}$ decreases to 1 , given the loss of their surface charge and particles agglomerate (Scheme 4(b)).

At $\mathrm{pH}$ values on either side of a neutral ( 3 and 10) compared to the limit values at $\mathrm{pH}=1$ and 12 , the inner ring is not visible because the amount of particles fixed in the first ring is not sufficient. The differences in spectra between modification of $\mathrm{pH}$ by addition of $\mathrm{HCl}$ or $\mathrm{C}_{6} \mathrm{H}_{8} \mathrm{O}_{7}$ are due to the difference between aggregated and agglomerated particles. In the case of $\mathrm{HCl}$, the metallic gold particles aggregate and the plasmon resonance presents a band of asymmetrical elongated particles higher than $600 \mathrm{~cm}^{-1}$ by UV-vis spectroscopy (Figure $3(\mathrm{~b})$ ). This band indicates that of the aggregate with visible interactions between the nanoparticles. For the $\mathrm{C}_{6} \mathrm{H}_{8} \mathrm{O}_{7}$ aperture, the resonance of each particle is preserved over the spectrum, despite the overall size of the aggregate. This indicates that the protective layer prevents the plasmon of the gold core particles from interacting and that the nanoparticles are agglomerated and not aggregated. Ulloa et al. [12] present a model in agreement with these observations as alternating charge density sign around the NPs, corresponding to electrical double layer.

To check the effect of the ionic state of the surrounding medium of the GNPs, $\mathrm{NaCl}$ has been added into a freshly prepared sol and CTLC tests have been performed on the sol after $30 \mathrm{~s}$ and 5 minutes (Figure 4). 30 seconds after $\mathrm{NaCl}$ addition, two colored circular boundaries are observed on CTLC test, a first one corresponds to the initial contact boundary (diameter $0.5 \mathrm{~cm}$ for a droplet of $20 \mu \mathrm{L}$ ) 
<smiles>[CH][C@@H](O)CC([O])(CC(=O)[O-])C(=O)O[Na]</smiles>

(a)

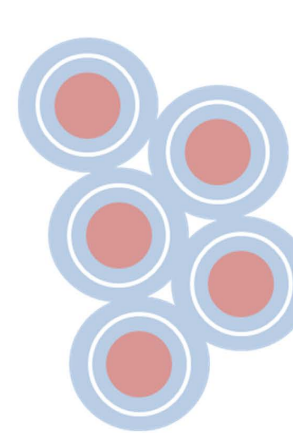

(b)
-Au-Au-Au-Au-<smiles>C=CC(=O)CC(CC(=O)OC)(C(=O)O)C(=O)O</smiles>

$\mathrm{H}_{3} \mathrm{O}^{+1} \mathrm{O}^{-} \mathrm{H}_{3} \mathrm{O}^{+}$<smiles>COC(=O)CC(O)(CC(=O)OC)C(=O)OC</smiles>

-Au-Au-Au-Au-

Scheme 4. Representation of a gold nanoparticle (pink color) surrounded by double layers of citrate (blue color) and the carboxylate functions involved in the protection of the nanoparticle after modification of $\mathrm{pH}$ by addition of citric acid (a) $\mathrm{pH}=3$ or (b) $\mathrm{pH}=1$.

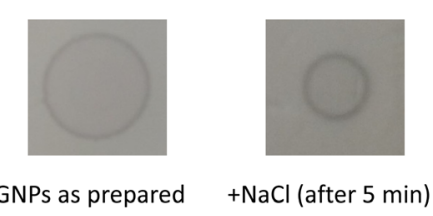

(a)

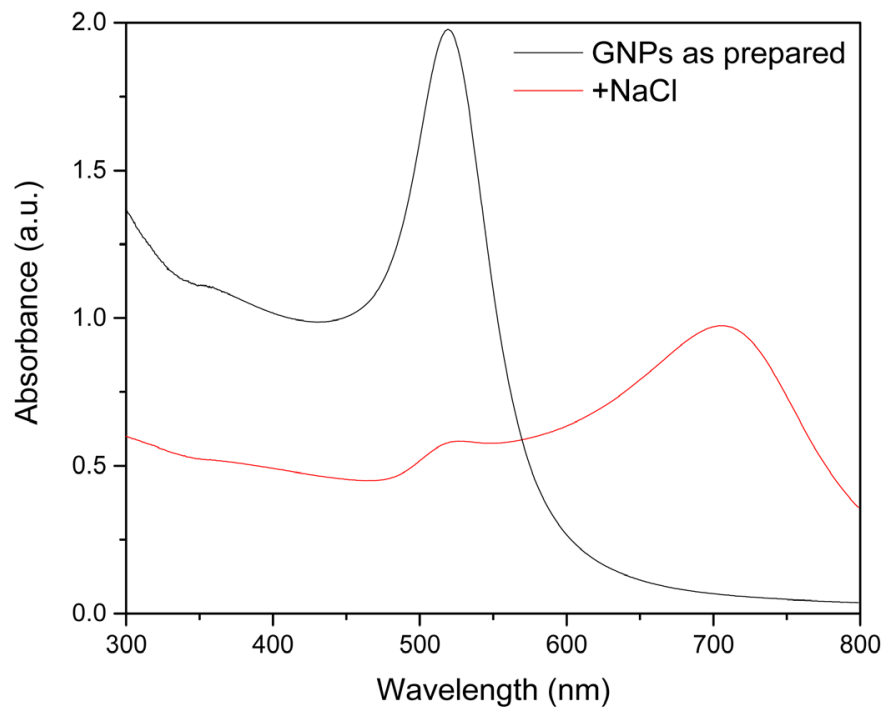

(b)

Figure 4. (a) CTLC profile during the kinetics of GNPs formation. (b) UV-vis spectra of gold nanoparticles as prepared and after addition of $\mathrm{NaCl}$ ( 5 min of contact).

and a second one to the moist-dry boundary (diameter $1 \mathrm{~cm}$ for the same droplet). The CTLC test of the same solution 5 minutes after the addition of $\mathrm{NaCl}$ displays a colored single circle (diameter $0.5 \mathrm{~cm}$ for a droplet of $20 \mu \mathrm{L}$ ) at the initial contact boundary. The UV-Vis spectrum evidences two surface plasmon resonances at $530 \mathrm{~nm}$ and $670 \mathrm{~nm}$, characteristic of asymmetrical elongated particles.

The change in the sol color from red (as prepared) to grey ( $5 \mathrm{~min}$ after $\mathrm{NaCl}$ addition) is due to the modification of particle interactions (Scheme 5). Indeed, 


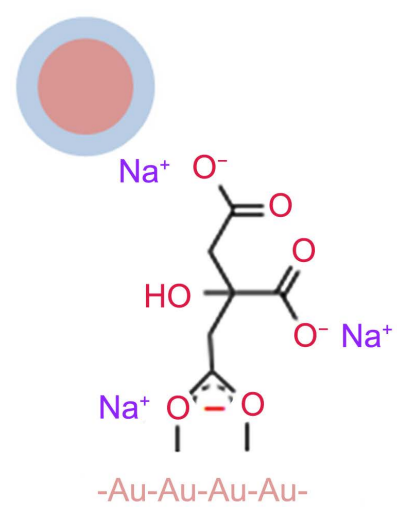

(a)

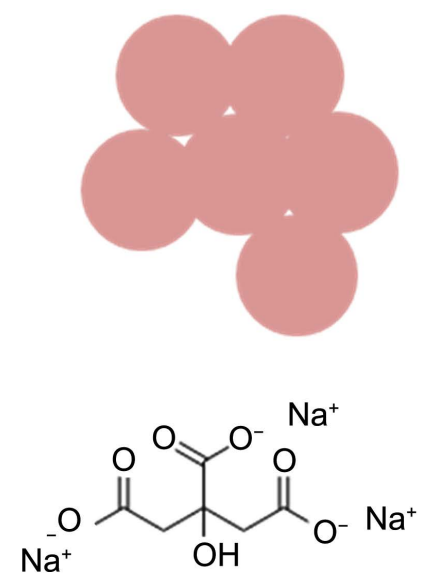

(b)

Scheme 5. Representation of a gold nanoparticle (pink color) surrounded by citrates (blue color) and the carboxylate functions involved by $\mathrm{NaCl}$ addition (a) $30 \mathrm{~s}$ later and (b) 5 min later: Aggregation of GNPs and formation of trisodium citrate.

sodium cations interact with the carboxylate functions altering the protection of the stabilizing citrate capsule. As a consequence, the gold nanoparticles aggregate.

SEM observations of the CTLC test of the initial contact boundary after $5 \mathrm{~min}$ of $\mathrm{NaCl}$ addition (Figure 5) show that the quasi totality of the matter is concentrated in the initial contact boundary. Typical SEM images clearly show huge aggregates of very small gold nanoparticles whose intrinsic size does not change (30 nm, DLS measure). In this case, the GNPs aggregates are unable to diffuse in the TLC and give a colored initial contact boundary and zone of CTLC.

The stability of GNPs sols before aggregation can vary from few days to months (for the most stable solutions). Before the sol presents a visible deposition, the stability can be lost. To show this fact and the advantages of following the sols by CTLC, gold nanoparticles in solution are presented by the comparison of "as prepared" (60 minutes) and after spontaneous (6 and 24 months) and induced (aggravated conditions of heat) aggregation. CTLC test allows a rapid response about the stability of nanoparticles and is really helpful for selecting batches for complementary control by DLS and TEM analyses.

The results of UV-vis spectra for aged GNPs' solution are presented in Figure 6. The profiles are not significantly changed with time only the intensity of the peak decreases with time and increases with heating. The plasmon band from UV-vis spectra and the color of the solutions do not reveal clearly the variation among fresh and agglomerated (6, 24 months and heated) GNPs' colloids.

At a scattering angle of $173^{\circ}$ (Figure 7), except the $\mathrm{NaCl}$ addicted one, all samples have a DLS signature of small diffusing particles, of about $30 \mathrm{~nm}$ for the fresh and the heated sample and higher than $70 \mathrm{~nm}$ for the aged samples. This result is consistent with UV-Vis absorption spectra. At a scattering angle of $13^{\circ}$, for which the contribution of large particles to DLS is enhanced, it is noticeable that the intensity of the scattered light by small particles is the dominant owned 


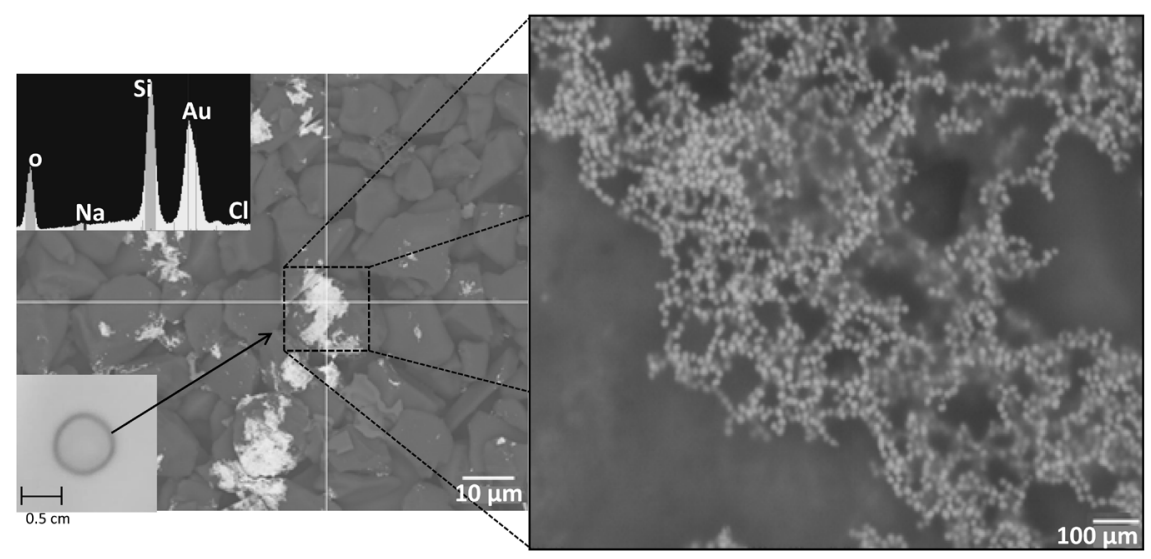

Figure 5. SEM images of the gold deposition on TLC (GNPs after 5 minutes of $\mathrm{NaCl}$ addition) and its zoom. EDX result corresponding to the area crossed.

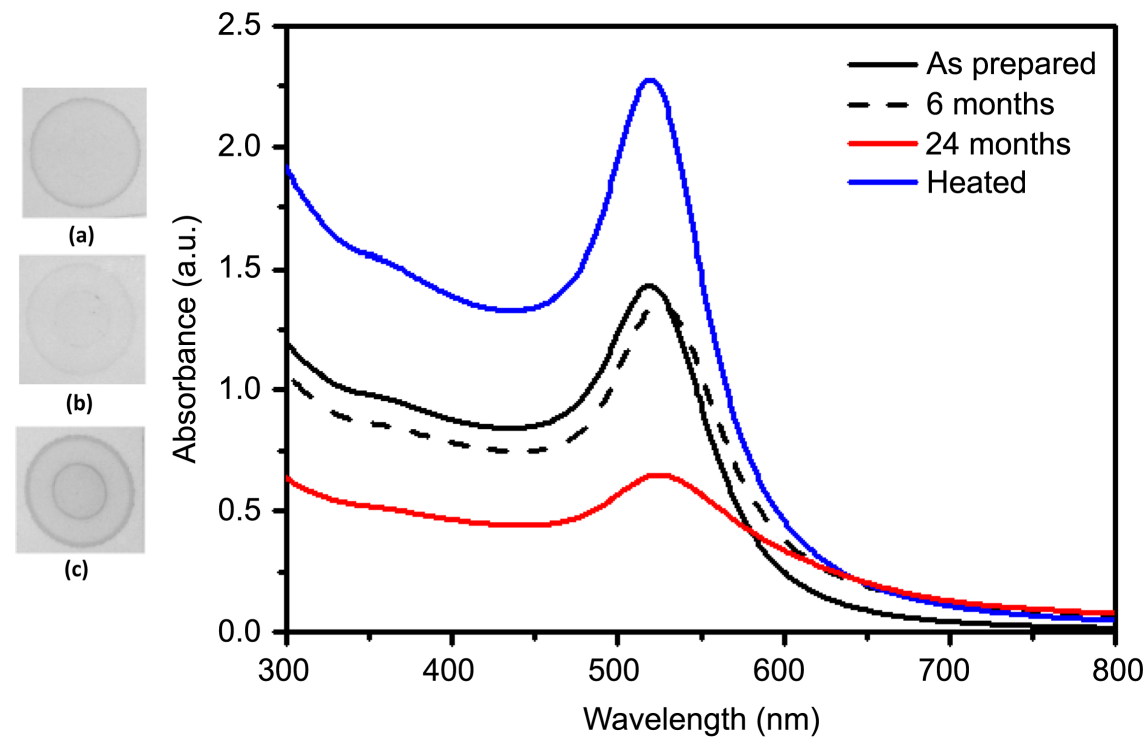

Figure 6. Evolution of CTLC and UV-vis spectra of colloidal sols with time and heating. (a) 6 months; (b) 24 months; (c) Heated.
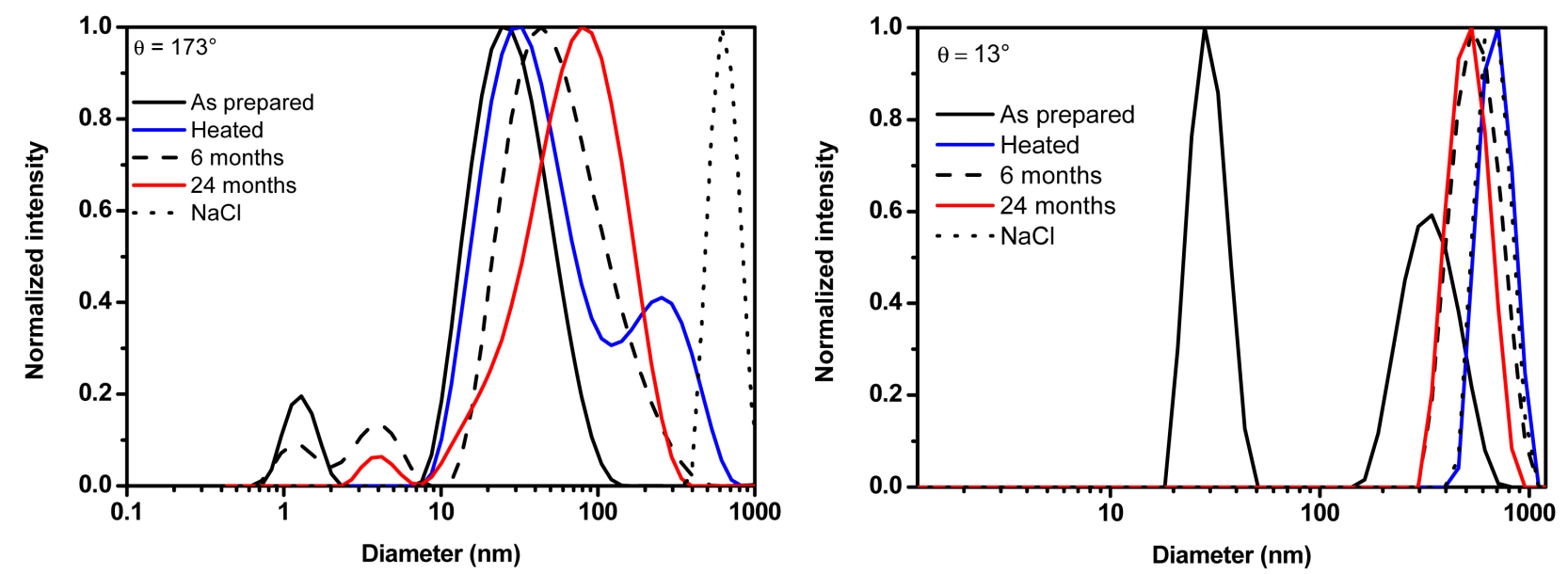

Figure 7. DLS of colloidal sols freshly prepared (60 minutes) and after ageing processes for both angles $\left(173^{\circ}\right.$ and $\left.13^{\circ}\right)$. 
in the freshly prepared sample while no traces of particles smaller than $500 \mathrm{~nm}$ contribute to the DLS of aged solutions. Moreover, as shown by UV-vis spectra, the absorbance at $633 \mathrm{~nm}$ which is the DLS laser wavelength is increased for aged samples with respect to the fresh one resulting into a decrease of the contribution of large particles to the DLS intensities. These results suggest that, except for the freshly prepared sample, all aged samples contain very large particle systems in a non-negligible amount. However, one has to keep in mind that the estimated diameters are hydrodynamic ones based on the assumption that the particles are spherical, which is not the form of aggregates as is revealed by Figure 8 and fractal systems are usually reported in the literature for gold.

The morphologies of gold colloids are observed with TEM technique by depositing the sol on the grid in Figure 9. TEM images of GNPs' colloid "as prepared" (Figure 9(a)) show nanoparticles independents or in small groups around $30 \mathrm{~nm}$. Aged colloids after 6 or 24 months and by heating (Figures 9(b)-(d)) contain small groups of NPs but do not show aggregates bigger than $200 \mathrm{~nm}$ as observed by DLS at $13^{\circ}$. The sample deposition onto a substrate to perform microscopic techniques may change the particle state and induce additional agglomeration or agglomeration rupture during and after drying [15]. TEM images may not deliver a reliable indication of gold agglomeration in solution. Gold colloid aggregated by $\mathrm{NaCl}$ contains only tridimensional groups of nanoparticles around $500 \mathrm{~nm}$ (Figure 9(e)).

GNPs' sol after 6 months presents a CTLC image similar to the colloid freshly prepared, Figure 10. The other images of CTLC for aged colloid solutions are different to those observed previously and the results are more complex. Two colored boundaries appear in Figure 4(b) and Figure 4(c): one in the same place as those previously observed and one inside this circle (placed about $1 / 2$ of the big diameter). GNPs display one large zone (ring) with a very red color and deep boundary, as well as one small inside disk with red color and a very light red boundary. It is inferred that great change occurs in colloid sols clearly visible with the CTLC test compared to UV-Visible and TEM. The limit of the size of
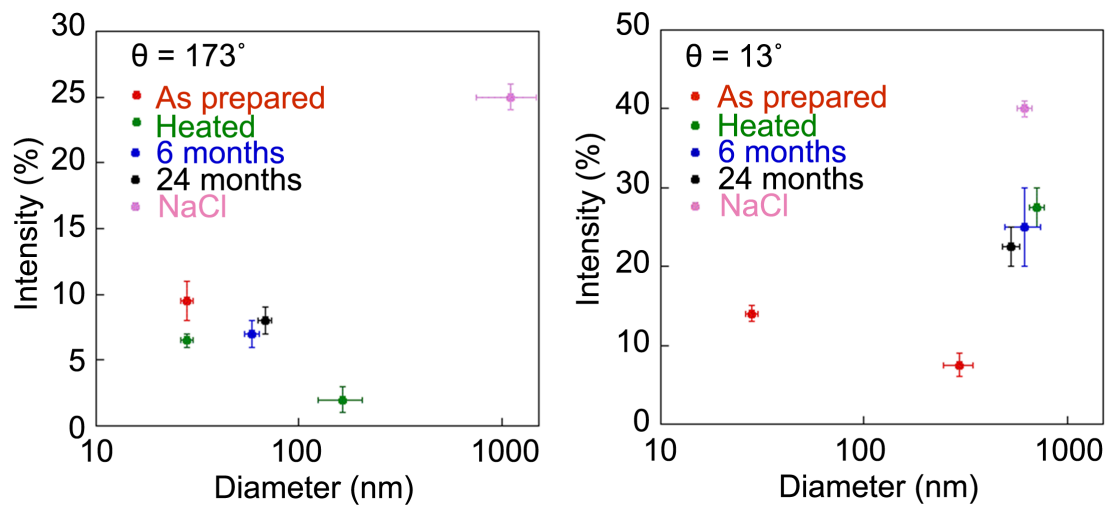

Figure 8. DLS intensities at scattering angles of $173^{\circ}$ and $13^{\circ}$. Comment: for the "As prepared" sol, as the intensity varies as diameter a power of six [9], we can estimate a number of small particles of about $2 \times 10^{6}$ that of the larger ones. 

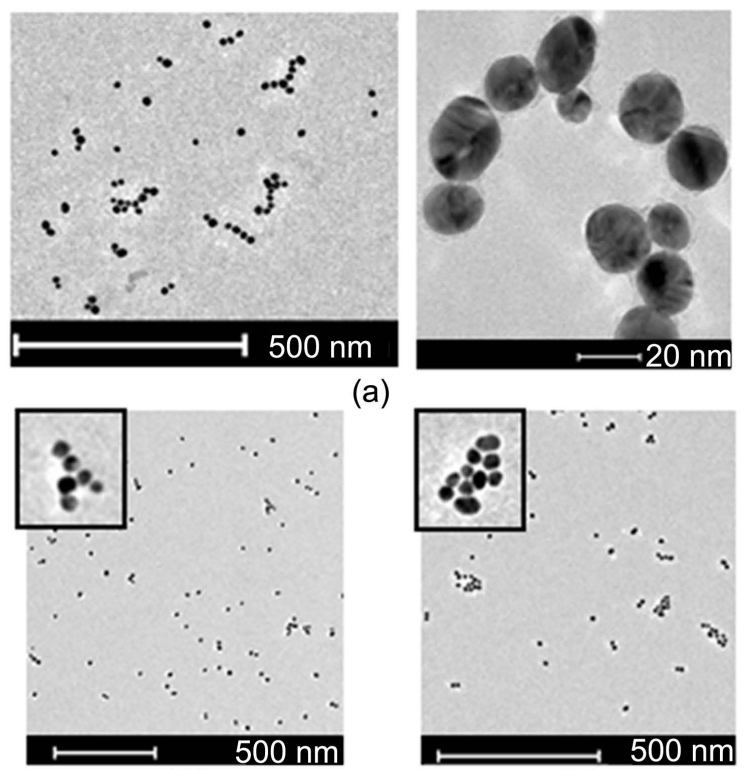

(a)

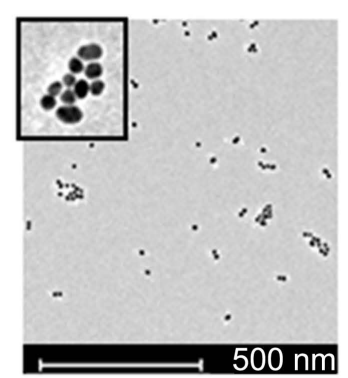

(b)

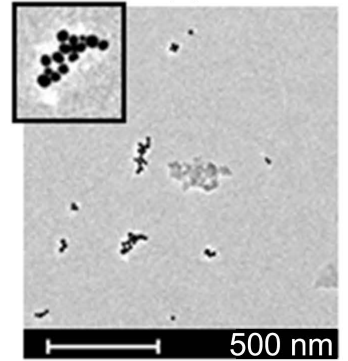

(d)

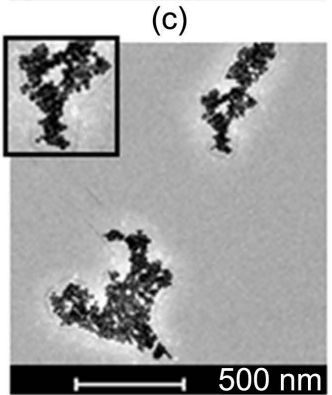

(e)

Figure 9. TEM micrographs of GNPs “as prepared” (60 minutes) (a), after 6 months (b), 24 months (c), heated (d) and $\mathrm{NaCl}$ addition (e).

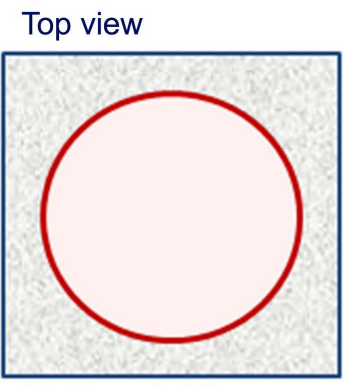

Stable sol of free nanoparticles

(a)

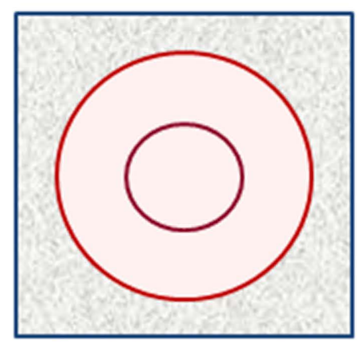

Partial aggregation

(b)

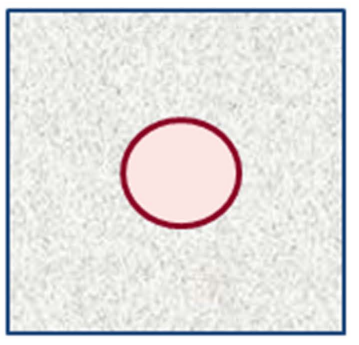

Large aggregates

(c)

Figure 10. The CTLC limit profiles obtained with spherical gold sols in water: (a) Visible particles on the moist-dry boundary; (b) Visible particles on the moist-dry boundary and the initial contact boundary; (c) Visible particles on the initial contact boundary and zone.

the aggregation system is not able to move far that the initial circle is estimated around $1 \mu \mathrm{m}$ regarding the texture of TL. This limit can change with the ligand (nature and charge). In the case of citrate, any gold nanoparticles adsorption on silica limit the migration on the plate. The water diffuses on the silica plate 
charged with the individual gold nanoparticles (or small agglomerates/aggregates) and transports them until the Moist-dry Boundary. The aggregated systems between about $1 \mu \mathrm{m}$ to visible particles (e.g. 24 months, heated or $\mathrm{NaCl}$-induced) present in the mixture stay on the initial contact zone, mainly an intense color is observable on the initial contact boundary (inside circle). In presence of one inside circle, we can conclude directly by Colloid Thin-Layer Chromatography: the bath must be discarded. If not, the batch can be used when the evaluation by DLS technique at $13^{\circ}$ shown major distribution for free particles.

Figure 10 summarizes the boundary profiles and corresponding CTLC observations for GNPs sols prepared by Turkevich's method. The fresh and stable sol shows free nanoparticles, mobile in the water drop, diffusing under the silica plate and concentrating on the boundary between the wet and dry boundary. The CTLC observation (Figure 10(a)) indicates that the sol is stable and can be considered for use. Unstable sols may exhibit CTLC profiles between Figure 10(b) with no deposition and no change in UV-visible spectra. The CTLC test (b) indicates that the nanoparticles are partially aggregated in solution. Visual precipitation in solution gives a profile with the borderline case being profile (Figure 10(c)). In this case, the deposit may fall directly onto the plate or form the coffee effect [16] with a deposit at the edge of the drop.

\section{Conclusion}

CTLC test is a new, easy and rapid method to control the production and conservation of handmade or commercial batches. The simple and inexpensive analysis on a silica plate is very useful to know and save the state of the solution. This test is useful for making screening with low utilization of sophisticated techniques. CTLC test is powerful when the UV profile of aged sol does not change significantly or the agglomerates/aggregates are greater than measure limits for DLS but not visible to a trained eye.

\section{Acknowledgements}

The authors would like to thank the China Scholarship Council (CSC) for the $\mathrm{PhD}$ grant during J. Luo's staying at the ICPEES Institut, University of Strasbourg. Valérie Caps, Christian Blanck, Marc Schmutz and Thierry Dintzer are thanked for helpful discussions.

\section{Conflicts of Interest}

The authors declare no conflicts of interest regarding the publication of this paper.

\section{References}

[1] Jans, H. and Huo, Q. (2012) Gold Nanoparticle-Enabled Biological and Chemical Detection and Analysis. Chemical Society Reviews, 41, 2849-2866.

https://doi.org/10.1039/C1CS15280G 
[2] Bessar, H., Venditti, I., Fratoddi, I., Benassi, L., Vaschieri, C., Azzoni, P., Pellacani, G., Magnoni, C., Botti, E., Casagrande, V., Federici, M., Costanzo, A., Fontana, L., Testa, G., Mostafa, F.F., Ibrahim, S.A. and Russo, M.V. (2016) Functionalized Gold Nanoparticles for Topical Delivery of Methotrexate for the Possible Treatment of Psoriasis. Colloids Surfaces B: Biointerfaces, 141, 141-147. https://doi.org/10.1016/j.colsurfb.2016.01.021

[3] Zhao, P., Li, N. and Astruc, D. (2013) State of the Art in Gold Nanoparticle Synthesis. Coordination Chemistry Reviews, 257, 638-665.

https://doi.org/10.1016/j.ccr.2012.09.002

[4] De Souza, C.D., Ribeiro Nogueira, B. and Rostelato, M.E.C.M. (2019) Review of the Methodologies Used in the Synthesis Gold Nanoparticles by Chemical Reduction. Journal of Alloys and Compounds, 798, 714-740. https://doi.org/10.1016/j.jallcom.2019.05.153

[5] de Melo, F.M., Klimuk Uchiyama, M., Nakamura, M., Kawassaki, R.K., Pelleschi Taborda, C. and Toma, H.E. (2019) Unravelling the Nature of the Spongy Dark Material in Aged Turkevich Gold Nanoparticles Colloidal Solutions by CytoViva Dark-Field Imaging and HRTEM Analysis. Micron, 121, 21-25. https://doi.org/10.1016/j.micron.2019.03.001

[6] Harvey, C.E., van Schrojenstein Lantman, E.M., Mank, A.J.G. and Weckhuysen, B.M. (2012) An Integrated AFM-Raman Instrument for Studying Heterogeneous Catalytic Systems: A First Showcase. Chemical Communications, 48, 1742-1744. https://doi.org/10.1039/c2cc15939b

[7] Zhao, B., Li, L., Yang, B., Yan, M., Zhai, Q. and Gao, Y. (2013) Structure Observation of Single Solidified Droplet by in Situ Controllable Quenching Based on Nanocalorimetry. Journal of Alloys and Compounds, 580, 386-391. https://doi.org/10.1016/j.jallcom.2013.06.110

[8] Liao, H.-G., Niu, K. and Zheng, H. (2013) Observation of Growth of Metal Nanoparticles. Chemical Communications, 49, 11720-11720. https://doi.org/10.1039/c3cc47473a

[9] Malvern Instruments Ltd. (2014) Zetasizer Nano Accessories Guide. 126 p. https://www.cif.iastate.edu/sites/default/files/uploads/Other_Inst/Particle\%20Size/A ccessories\%20and\%20Cells\%20Guide.pdf

[10] Hu, M., Chen, J., Li, Z.-Y., Au, L., Hartland, G.V., Li, X., Marquez, M. and Xia, Y. (2006) Gold Nanostructures: Engineering Their Plasmonic Properties for Biomedical Applications. Chemical Society Reviews, 35, 1084-1094. https://doi.org/10.1039/b517615h

[11] Yu, Y., Chen, Z., He, S., Zhang, B., Li, X. and Yao, M. (2014) Direct Electron Transfer of Glucose Oxidase and Biosensing for Glucose Based on PDDA-Capped Gold Nanoparticle Modified Graphene/Multi-Walled Carbon Nanotubes Electrode. Biosensors and Bioelectronics, 52, 147-152. https://doi.org/10.1016/j.bios.2013.08.043

[12] Franco-Ulloa, S., Tatulli, G., Bore, S.L., Moglianetti, M., Pompa, P.P., Cascella, M. and De Vivo, M. (2020) Dispersion State Phase Diagram of Citrate-Coated Metallic Nanoparticles in Saline Solutions. Nature Communications, 11, Article No. 5422. https://doi.org/10.1038/s41467-020-19164-3

[13] Grys, D.-B., de Nijs, B., Salmon, A.R., Huang, J., Wang, W., Chen, W.-H., Scherman, O.A. and Baumberg, J.J. (2020) Citrate Coordination and Bridging of Gold Nanoparticles: The Role of Gold Adatoms in AuNP Aging. ACS Nano, 14, 8689-8696. https://doi.org/10.1021/acsnano.0c03050

[14] Al-Johani, H., Abou-Hamad, E., Jedidi, A., Widdifield, C.M., Viger-Gravel, J., San- 
garu, S.S., Gajan, D., Anjum, D.H., Ould-Chikh, S., Hedhili, M.N., Gurinov, A., Kelly, M.J., El Eter, M., Cavallo, L., Emsley, L. and Basset, J.M. (2017) The Structure and Binding Mode of Citrate in the Stabilization of Gold Nanoparticles. Nature Chemistry, 9, 890-895. https://doi.org/10.1038/nchem.2752

[15] Zimbone, M., Calcagno, L., Messina, G., Baeri, P. and Compagnini, G. (2011) Dynamic Light Scattering and UV-vis Spectroscopy of Gold Nanoparticles Solution. Materials Letters, 65, 2906-2909. https://doi.org/10.1016/j.matlet.2011.06.054

[16] Deegan, R.D., Bakajin, O., Dupont, T.F., Huber, G., Nagel, S.R. and Witten, T.A. (1997) Capillary Flow as the Cause of Ring Stains from Dried Liquid Drops. Nature, 389, 827-829. https://doi.org/10.1038/39827 